\title{
Cross-sectional analysis of tubular polymer semi-finished products using ultrasound in comparison with other measuring methods
}

\begin{abstract}
In the sector of biomedical engineering and implant technology, high-precision geometry is often decisive for successful end product functionalization. Especially in the production of tubular polymer semi-finished products, e.g. for stent fabrication, it is important to assure the desired parameters, such as inner and outer diameter and wall thickness. Within the current study we analyzed semifinished products for manufacturing of polymeric stents using three different methods. Biodegradable poly-L-lactide (PLLA) tubes were examined by means of micro computed tomography, ultrasonic scanning and scanning electron microscopy. The final evaluation presents clear advantages of the ultrasonic measuring method for the measurement of outer and inner diameter and wall thickness.
\end{abstract}

Keywords: micro computed tomography, ultrasonic measurement, extrusion, PLLA, tubular semi-finished product, scanning electron microscopy

https://doi.org/10.1515/cdbme-2020-3039

\footnotetext{
*Corresponding author: Olga Sahmel, Institute for Biomedical Engineering, Rostock University Medical Center, FriedrichBarnewitz-Str. 4, 18119 Rostock, Germany, e-mail: olga.sahmel@uni-rostock.de

Stefan Siewert: Institute for ImplantTechnology and Biomaterials e.V., Friedrich-Barnewitz-Str. 4, 18119 Rostock-Warnemünde Wolfram Schmidt: Institute for Biomedical Engineering, Rostock University Medical Center Rostock, Friedrich-Barnewitz-Str. 4, 18119 Rostock, Germany

Klaus-Peter Schmitz: Institute for ImplantTechnology and Biomaterials e.V., Institute for Biomedical Engineering, FriedrichBarnewitz-Str. 4, 18119 Rostock-

Warnemünde

Niels Grabow: Institute for Biomedical Engineering, Rostock University Medical Center Rostock, Friedrich-Barnewitz-Str. 4, 18119 Rostock, Germany
}

\section{Introduction}

Tubes, cannulae, catheters as well as tubular implants made of polymers are of great importance in modern medicine. Regardless of whether they are invasive or non-invasive, tubular medical devices contribute to life-saving measures. The requirements are manifold and increase with degree of invasiveness. In addition to basic requirements, such as biocompatibility, there are high demands with regard to geometry and mechanical properties.

The morphological investigations of the cross-sectional geometry are often only carried out selectively and not always non-destructively. Furthermore, measurement of outer and inner diameter, as well as wall thickness, requires additional work steps, respectively.

Within the current study we analyzed semi-finished products for manufacturing of polymeric stents using three different methods. Based on earlier work, an ultrasonic measuring method is evaluated as an alternative to micro computed tomography and scanning electron microscopy for the analysis of polymer microtubes $[1,2]$.

\section{Materials and Methods}

\subsection{Extrusion}

Extrusion was conducted using a HAAKE MiniLab II (Thermo Fisher Scientific, Karlsruhe, Germany) extruder with contra-rotating twin screws. A Force Feeder device (Thermo Fisher Scientific, Karlsruhe, Germany) was used for granulate transport. A 2-axis laser scanner (ZUMBACH Elektronik, Orpund, Switzerland) was used to monitor the outer diameter of the extrudate during the extrusion process.

A mounted nozzle with an external and internal diameter of $2.30 \mathrm{~mm}$ and $1.75 \mathrm{~mm}$ served for the raw-form modulation of the extrudate. A discharge conveyor (Thermo Fisher Scientific, Karlsruhe, Germany) was placed near the nozzle 
and allows for desired cross-sectional geometry by velocity regulation. The extrusion parameters used are summarized in Table 1. Medical grade PLLA Resomer L210 (Evonik Industries, Germany) was used [2].

Table 1: Setting parameters of the extrusion process PLLAResomer L210

\begin{tabular}{ll}
\hline Material & Extruder setting parameters \\
\hline PLLA-Resomer L210 & $T_{E}=210^{\circ} \mathrm{C}, n_{E}=561 / \mathrm{min}$ \\
& $T_{D}=225^{\circ} \mathrm{C}, n_{A}$ - optional \\
& $T_{F F}=16^{\circ} \mathrm{C}, n_{F F}=40 \%$ \\
\hline
\end{tabular}

$T_{E}-$ Temperature of the extruder, $n_{E}-$ Rotation speed of extruder screws,

$T_{D}$ - Temperature of the nozzle, $n_{A}$ - Haul-off speed

$T_{F F}-$ Temperature of the force feeder, $n_{F F}-$ Power of force feeder

For morphological investigations, three samples with a length of approx. $8 \mathrm{~cm}$ were randomly selected. The outer diameter of sample 1, 2 and 3 being approx. $1 \mathrm{~mm}, 2 \mathrm{~mm}$ and $2 \mathrm{~mm}$, respectively.

\subsubsection{Micro computed tomography}

The cross-sectional analysis of the extruded specimens was carried out by means of micro computed tomography ( $\mu-\mathrm{CT}$, Bruker SKYSCAN 1172, Kontich, Belgium). The scanning process is performed with a $360^{\circ}$ movement of the sample around its own longitudinal axis. All samples were scanned without filters with a source voltage of $80 \mathrm{kV}$ and $100 \mu \mathrm{A}$ current. Further sample specific settings are summarized in table 2 .

Table 2: Setting parameters of the $\mu-C T$ scanning process

\begin{tabular}{llll}
\hline $\boldsymbol{\mu}$-CT & Sample 1 & Sample 2 & Sample 3 \\
\hline Image Pixel Size $[\mu \mathrm{m}]$ & 4.88 & 6.89 & 20.32 \\
\hline Number of Columns & 1280 & 640 & 640 \\
\hline Number of Rows & 1024 & 512 & 512 \\
\hline
\end{tabular}

The samples were evenly divided into five segments, in which one cross-sectional image was analyzed, respectively. Wrought plasticine was used for marking, which served for the visibility in $\mu-\mathrm{CT}$ (Figure 1).

Based on the five cross-sectional images per sample, the determination of the outer and inner diameters as well as the wall thickness over the complete circumference was carried out in $5^{\circ}$ increments. The measurement is based on the segmentation of the digital cross-sectional image by entering the respective pixel size (see Table 2). The software automatically calculates the values sought from the ratio of pixel number to pixel size.

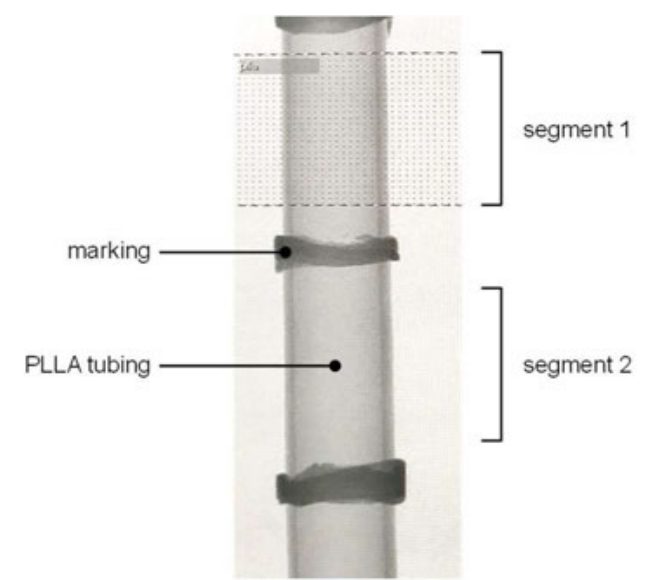

Figure 1: $\mu-C T$ screen shot during scanning one of five segments

\subsubsection{Ultrasonic measurement}

The measurement was carried out using an UlraScan 1012, in a version with four sensors and $20 \mathrm{MHz}$ transducer frequency from NDC Technologies Limited (Maldon, United Kingdom). Measurements were carried out in water. It is important to ensure that the tubular sample remains watertight at both ends. Air inside the tubular sample represents a mandatory requirement for the measurement. The calibration to the polymer used is based on a sample of the same material with known wall thickness. This was determined in advance in $\mu-\mathrm{CT}$ and entered during calibration by UltraScan. Consequently, the instrument automatically determines material-specific ultrasonic speed with which it can analyze unknown samples of the same material regarding inner and outer diameter and wall thickness. Another important aspect is the centric alignment of the sample in the measuring field. Mean values of the specimen diameter and wall thickness per measuring point are calculated from four sensor data. Referred to manufacturer's specifications, outer diameter ranging between $0.025-12 \mathrm{~cm}$ can be measured. Analogous to $\mu-\mathrm{CT}$ analysis, the measurement was performed at the same five segment points of each sample.

\subsubsection{Scanning electron microscopy}

Scanning electron microscopy (SEM) (QuattroS, Thermo Fisher Scientific, Waltham, USA) was used for crosssectional analysis. SEM was deliberately chosen as the final examination because of the preparation procedure. Here, an 
approximately $1-2 \mathrm{~mm}$ long sample was cut out from each of the five segments of the specimen with a scalpel and fixed on a specimen holder. It is important to ensure that the crosssectional plane remains as straight as possible. This facilitates focusing on the cutting plane and thus the manual measurement of inner and outer diameter and wall thickness, $(n=4)$ respectively. The analysis was performed uniformly for all samples in low vacuum mode at $51 \mathrm{~Pa}$ and an excitation voltage of $5.00 \mathrm{kV}$. The magnification is based on maximum and complete display of the cross-section on the monitor.

\section{Results and Discussion}

The extrusion process resulted in tubular polymer semifinished products with an outer diameter in the range of 0.6 $2.3 \mathrm{~mm}$ within a short period of time and without extensive preparation and post-processing.

Scanning and reconstruction using $\mu-\mathrm{CT}$ analysis proved to be much more difficult with the thinner specimen (sample 1) than with specimen 2 and 3. Due to the $360^{\circ}$ movement, minimal vibration resulted in poor cross-sectional acquisition, see Figure 2a. After several repetitions, the assumed quality of the cross-sectional image was finally achieved (Figure 2b).
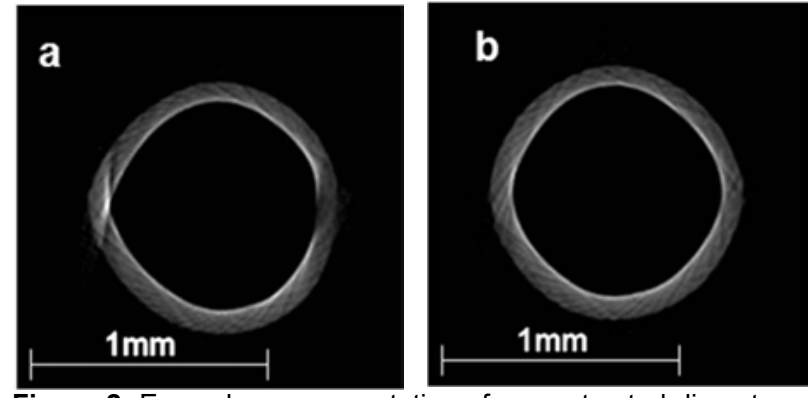

Figure 2: Exemplary representation of reconstructed diameter of sample 1 with direct comparison defective (a) and required (b) quality

A calibration of the ultrasonic measuring instrument on PLLA material allows for a fast measurement, especially for samples 2 and 3. The sample holder was not optimally suited for sample 1 . The centring of the sample with respect to the measuring transducer turned out to be laborious and thus more time-consuming. Although the sample is within the range of the manufacturer's specifications, an alternative holder will need to be considered.

Figure 3 shows examples of how the outer diameter (Figure 3a), the inner diameter (Figure 3b) and the wall thickness (Figure 3c) were measured. Exact measurement of cross-sections in SEM images was challenging with regard to manual selection of cutting edges, resulting from manual sample preparation (see Figure 3).

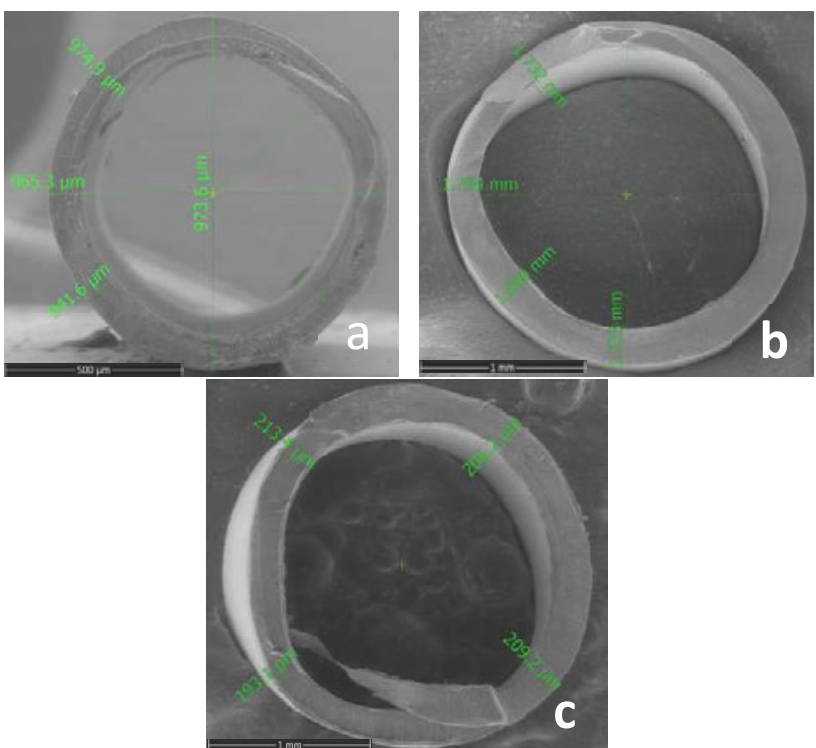

Figure 3: Exemplary representation of SEM cross-sections with the measurement of: a) outer diameter, sample 1; b) inner diameter, sample 2; c) wall thickness, sample 3

The results of the three measuring methods for the respective sample are summarized in Table 3 . The mean values are based on $n=5$ measuring positions per sample.

Table 3: Results of the outer diameters $(O D)$, inner diameters (ID) and wall thicknesses $(W T)$ of the three measuring methods in direct comparison for each sample, mean value \pm standard deviation, $n=5$

\begin{tabular}{llll} 
Sample 1 & $\boldsymbol{O D}[\mathrm{mm}]$ & $\boldsymbol{I}[\mathrm{mm}]$ & $\boldsymbol{W}[\mathrm{mm}]$ \\
\hline$\mu$-CT & $0.905 \pm 0.018$ & $0.763 \pm 0.019$ & $0.071 \pm 0.003$ \\
Ultrasonic & $0.908 \pm 0.028$ & $0.704 \pm 0.025$ & $0.102 \pm 0.003$ \\
SEM & $0.929 \pm 0.026$ & $0.738 \pm 0.018$ & $0.096 \pm 0.004$ \\
Sample 2 & & & \\
$\mu$-CT & $2.113 \pm 0.057$ & $1.728 \pm 0.048$ & $0.192 \pm 0.011$ \\
Ultrasonic & $2.116 \pm 0.048$ & $1.750 \pm 0.039$ & $0.183 \pm 0.007$ \\
SEM & $2.103 \pm 0.035$ & $1.708 \pm 0.037$ & $0.217 \pm 0.008$ \\
Sample 3 & & & \\
$\mu$-CT & $2.057 \pm 0.028$ & $1.692 \pm 0.022$ & $0.182 \pm 0.005$ \\
Ultrasonic & $2.295 \pm 0.032$ & $1.932 \pm 0.024$ & $0.182 \pm 0.005$ \\
SEM & $2.089 \pm 0.031$ & $1.686 \pm 0.045$ & $0.211 \pm 0.008$
\end{tabular}

Basically, the values of all three measuring methods are similar. The values of the ultrasound with $O D=2.295 \pm 0.032 \mathrm{~mm}$ and $I D=1.932 \pm 0.024 \mathrm{~mm}$ appear remarkably large in comparison to $\mu-\mathrm{CT}$ and SEM for sample 3. The difference of approx. $0.2 \mathrm{~mm}$ nevertheless 
appears to be correct, because sample 3 was already optically slightly larger than sample 2 . Thus, the three values presented for $O D$ of sample 2 remain credible. Nevertheless, $I D$ and $W T$ also contribute to the overall evaluation.

The results of the measurements of the wall thicknesses for sample 2 are very similar in the $\mu$-CT and ultrasonic method, and even identical for sample 3. In contrast, the results of the measurements in the SEM images method for samples 2 and 3 differ considerably. The SEM method is therefore described as less reliable in this context.

For sample 1, on the other hand, the SEM value for the outer diameter with $O D=0.929 \pm 0.026 \mathrm{~mm}$ differs from $\mu$ $\mathrm{CT}$ and ultrasound. Compared to $\mu-\mathrm{CT}$ and ultrasound, the SEM values for the inner diameter $(I D=0.738 \pm 0.018 \mathrm{~mm})$ and wall thickness $(W T=0.096 \pm 0.004 \mathrm{~mm})$ are in the middle range of the three measuring methods. With $O D=0.905 \pm 0.018 \mathrm{~mm} \quad$ for $\quad \mu-\mathrm{CT} \quad$ and $O D=0.908 \pm 0.028 \mathrm{~mm}$ for ultrasound, the values for the outer diameter are almost identical. This leaves the SEM results somewhat questionable. On the other hand, the values for the inside diameter for $\mu-C T(I D=0.763 \pm 0.019 \mathrm{~mm})$ and ultrasound $(I D=0.704 \pm 0.025 \mathrm{~mm})$ differ greatly from each other and form the maximum and minimum values. In contrast, the values for the wall thickness represent the minimum value for $\mu$-CT $(W T=0.071 \pm 0.003 \mathrm{~mm})$ and the maximum value for ultrasound $(W T=0.102 \pm 0.003 \mathrm{~mm})$.

A standard deviation occurs only at the second decimal place for diameters and only at the third decimal place for wall thickness.

As all investigated methods appear mutually supplemental, no single preferred method was derived from this series of measurements.

\section{Conclusion}

The investigation shows that the applied methods $\mu-C T$, ultrasound and SEM are suitable for the measurement of outer and inner diameters and wall thicknesses of tubular semi-finished products made of polymer.
Characterization via $\mu-\mathrm{CT}$ represents a non-destructive method. An unstable specimen position with filigree specimens potentially leads to artifacts in cross-sectional images. Therefore, stable sample positioning and particularly the high expenditure of time represent the major limitations of this method.

Measurements using the SEM method cannot be carried out non-destructively. Furthermore, both the preparation and the manual measurement of cross-sectional images are timeconsuming and lead to systematic errors.

In ultrasonic measurements, there is a minor influence of systematic errors compared with $\mu$-CT and SEM methods. In addition, ultrasonic measurement can be performed nondestructively and in a time-saving manner. In the future, an improved sample-holder for the measurement of filigree samples will be constructed, and will allow for a fine adjustment of samples in the measuring field.

In summary, characterization of tubular polymeric specimens by means of the presented ultrasonic method represents a cheap and promising alternative for precise and high throughput quality control.

\section{Author Statement}

Research funding: Financial support by the Federal Ministry of Education and Research (BMBF) within RESPONSE "Partnership for Innovation in Implant Technology". Conflict of interest: Authors state no conflict of interest. Informed consent: Informed consent is not applicable.

\section{References}

[1] Sahmel O, Optimization of manufacturing processes for biodegradable polymeric stents regarding improved mechanical properties. Current Directions in Biomedical Engineering; 2018; 4(1): 583-585

[2] Sahmel O, Extrusion as a manufacturing processe for polymer micro-tubes for various bio-medical applications. Berlin: Current Directions in Biomedical Engineering; 2019; 5(1): 489-492 